\title{
Magnetized plasma sheath with two positive ions where collision frequencies have a power law dependency on ions velocities
}

\author{
S. Farhad Masoudi ${ }^{1} \cdot$ Mansor Khoramabadi $^{2}$
}

Received: 10 June 2015/Accepted: 18 August 2015/Published online: 7 September 2015

(c) The Author(s) 2015. This article is published with open access at Springerlink.com

\begin{abstract}
We study the dynamics of collisional magnetized plasma sheath with two species of positive ions by using the plasma fluid model. The basic equations of the fluid model are solved numerically where the sheath is in the external magnetic field and the elastic collision between ions and neutrals has been taken into account. In our model, we assume that the collisional momentum transferring cross section has a power law dependency on ion flow velocity. Our analysis demonstrates that the sheath dynamics are sensitive to the power law dependency, especially for the ion with greater density.
\end{abstract}

Keywords Magnetized plasma sheath - Multi-component plasma $\cdot$ Ion-neutral collision

\section{Introduction}

The understanding of sheath formation of a plasma in contact with an absorbing wall is one of the oldest problems in plasma physics. However, the study of the plasma sheath is still one of the interests in plasma [1-9], since it plays an important role in many plasma applications such as processing and fabrication of semiconductor devices [10], modification of the chemical and physical properties of the materials [11], film deposition [12, 13], etching [14,

S. Farhad Masoudi

masoudi@kntu.ac.ir

1 Department of Physics, K.N. Toosi University of Technology, P.O. Box 15875-4416, Tehran, Iran

2 Department of Physics, Boroujerd Branch, Islamic Azad University, Boroujerd, Iran
15], ion extraction [16], sputtering [17, 18], and modal and discrete focusing $[19,20]$.

In the sheath region, the ions are accelerated by the electric field and implanted on the absorbing wall. Therefore, understanding the dynamic properties of the plasma sheath is imperative for proper control over the implantation processing. Hence, many theoretical and experimental researches have been developed to study the plasma sheath structure [21-26].

External magnetic field and collisions between ions and neutrals are two important components that can affect the plasma sheath dynamics. Many works have investigated the collisional and magnetized plasma sheath containing ion and electron. Besides the works studying two-component plasma sheath composed of ion and electron, the investigation of multi-component plasma sheath has recently drawn considerable attention [22-39]. For example, experimental investigation of plasma sheath containing two positive ions can be found in Refs. [34-37]. References [34] and [35] have investigated the plasma sheath containing two positive ions, $\mathrm{Ar}^{+}$ and $\mathrm{He}^{+}$, experimentally, using dc hot-filament multidipole plasma system. Experimental study of two-ion species argonxenon plasma can be found in Refs. [36, 37] and [36, 37].

Besides experimental investigations, there exist related theoretical studies on multi-component plasma sheath. For instance, the plasma including hot electrons and multiply charged ions has been investigated in Ref. [38]. In Ref. [39], the effect of positive ion temperature on magnetized electronegative sheath in low-pressure argon-oxygen plasma mixture has been studied. Multi-component non-magnetized and magnetized plasmas containing electron and two positive ions have been analyzed in Refs. [26] and [32], respectively.

Investigation of the effect of collisions between ions and neutrals on plasma sheath dynamics is more important in magnetized plasma sheath, since it can diminish the effects 
of magnetic force on sheath dynamics. For example, Refs. [40] and [33] have reported this effect in two-component and multi-component plasma sheaths, respectively.

In Ref. [33], the effects of ion-neutral collision on the characteristics of a magnetized plasma sheath composed of two species of positive ions have been investigated. Three assumptions were taken into consideration in Ref. [33] as follows: (1) the ion-neutral collision cross section is constant, (2) the ion-neutral collision frequency depends on ion velocity in the cathode direction, and (3) it has been assumed that the ions enter into the sheath vertically. However, the momentum transfer cross section of ion-neutral collision is generally a function of velocity (see chapter 3 of Ref. [41]). Although two specific cases have been investigated in literature (constant mean free path and constant ion mobility), however, the dependency of ion-neutral collision cross section on ion velocity depends on plasma characteristics such as pressure, ions in plasma and the range of their energies.

In general, the ion-neutral collision cross section $\sigma$ and frequency $v$ have a power law dependency on the ion speed $v$, as follows [42, 43]:

$\sigma=\sigma_{\mathrm{s}}\left(\frac{v}{c_{\mathrm{s}}}\right)^{p}$

$v=n_{\mathrm{n}} \sigma_{\mathrm{s}} c_{\mathrm{s}}\left(\frac{v}{c_{\mathrm{s}}}\right)^{p+1}=n_{\mathrm{n}} v \sigma_{\mathrm{s}}\left(\frac{v}{c_{\mathrm{s}}}\right)^{p}=n_{\mathrm{n}} v \sigma$,

where $n_{\mathrm{n}}$ is the neutral gas density, $c_{\mathrm{s}}$ is the ion acoustic velocity, $\sigma_{\mathrm{s}}$ is the ion collision cross section measured at ion acoustic velocity, and ' $p$ ' is a dimensionless parameter that ranges from 0 to -1 . For example, the plasma sheath at low and medium pressures is examined by constant cross section $(p=0)$; the constant collision frequency for momentum transfer $(p=-1)$ is considered for higher pressures, and for argon plasma in the range of $3-300 \mathrm{eV}, p$ is -0.25 . Many works have investigated the effects of power law dependency on two-component or electronegative plasma sheath such as Refs. [43-45]. From here, we name the power law dependency of collision cross section to ion velocity as collisional regime between ions and neutrals.

In this article, we modified the results of Ref. [33] by considering the power law dependency between collision cross section and ion velocity. Also, in our model, the collisions frequency depends on ion velocity and not on its vertical component. It leads to increasing the effects of collision. Although these two cases approximately lead to the same ion dynamics in two-component magnetized plasma sheath [46], our study shows that they lead to different results in multi-component magnetized plasma sheath. In addition, we consider oblique entrance of the ions into the sheath as a result of magnetic presheath.

The layout of the article is as follows. In "Fluid model and basic equations", we present the model and its equations for studying the magnetized collisional plasma sheath composed of electron and two positive ions with different masses. "Fluid model and basic equations" ends with the presentation of the normalized form of the basic equations. In "Numerical results and discussion", we report our numerical results and analyze their implications. "Conclusions" concludes the article by a summary and outlook of our finding.

\section{Fluid model and basic equations}

In the presence of a magnetic field, the ions enter into the sheath with an oblique entrance angle as a result of a magnetic presheath. To find the entrance angle of ions into the sheath, it is necessary to solve the fluid equations in the presheath exactly. However, to avoid complex mathematical techniques, the fluid equations in the plasma sheath can be investigated by considering the Bohm criterion at the sheath boundary. Following some theoretical works used in this approach (e.g., [47-52]), we consider a magnetized plasma sheath having one-dimensional coordinate space and three-dimensional velocity space. Also, we consider a Cartesian coordinate where the $z$-direction is chosen as the depth direction (normal to the wall). The plasma sheath boundary is located at $z=0$, and the external magnetic field is embedded in the $x-z$ plane and makes $\theta$ angle with respect to the $z$-direction. As a result of the magnetic field, the plasma-wall transition consists of a magnetic presheath followed by a Debye sheath. In this article, we study the Debye sheath in which the neutrality is broken.

We assume that the plasma consists of thermal electrons and two positive cold ions with different masses. We suppose that the energy of the electrons is sufficiently low. It enables us to neglect the ionization in the plasma sheath. We explain the basic equations of the model using the following variables:

\begin{tabular}{llll}
\hline Variables & Local value & Boundary value & $\begin{array}{l}\text { Normalized } \\
\text { form }\end{array}$ \\
\hline $\begin{array}{l}\text { Electron density } \\
\text { Electrostatic } \\
\text { potential }\end{array}$ & $n_{\mathrm{e}}$ & $n_{\mathrm{e} 0}$ & $N_{\mathrm{e}}=n_{\mathrm{e}} / n_{\mathrm{e} 0}$ \\
$\begin{array}{l}\text { Ion velocity } \\
\text { v }\end{array}$ & $\boldsymbol{v}_{1}=\left(v_{1 x}, v_{1 y}\right.$, & $\boldsymbol{v}_{10}=\left(v_{1 x 0}, v_{1 y 0}\right.$, & $\boldsymbol{u}_{1}=\boldsymbol{v}_{1} / c_{s 1}$ \\
& $\left.v_{1 z}\right)$ & $\left.v_{1 z 0}\right)$ & \\
& $\boldsymbol{v}_{2}=\left(v_{2 x}, v_{2 y}\right.$, & $\boldsymbol{v}_{20}=\left(v_{2 x 0}, v_{2 y 0}\right.$, & $\boldsymbol{u}_{2}=v_{2} / c_{s 1}$ \\
& $\left.v_{2 z}\right)$ & $\left.v_{2 z 0}\right)$ & \\
Ion speed & $v_{1}$ & $v_{10}$ & $u_{1}=v_{1} / c_{s 1}$ \\
& $v_{2}$ & $v_{20}$ & $u_{2}=v_{2} / c_{s 1}$ \\
Ion density & $n_{1}$ & $n_{10}$ & $N_{1}=n_{1} / n_{\mathrm{e} 0}$ \\
& $n_{2}$ & $n_{20}$ & $N_{2}=n_{2} / n_{\mathrm{e} 0}$ \\
Depth direction & $z$ & 0 & $\xi=z / \lambda_{D}$ \\
\hline
\end{tabular}


where $T_{\mathrm{e}}$ is the electron temperature, $c_{s j}=\left(k_{\mathrm{B}} T_{\mathrm{e}} / m_{j}\right)^{0.5}$ is the ion acoustic speed for the $j$-th ion $\left(j=1\right.$ and 2), $m_{j}$ is the mass of the $j$-th ion, $\lambda_{\mathrm{D}}=\left(\varepsilon_{0} k_{\mathrm{B}} T_{\mathrm{e}} / n_{\mathrm{e} o} e^{2}\right)^{0.5}$ is the electron Debye length and $k_{\mathrm{B}}$ is the Boltzmann constant. Using these definitions, the basic equations of the plasma sheath according to the fluid model are as follows:

Continuity equation of $j$-th ion:

$\vec{\nabla} \cdot\left(n_{j} \vec{v}_{j}\right)=0$.

Motion equation of the $j$-th ion:

$m_{j}\left(\vec{v}_{j} . \vec{\nabla}\right) \vec{v}_{j}=-e \vec{\nabla} \varphi+e \vec{v}_{j} \times \vec{B}-m_{j} v_{j} \vec{v}_{j}$,

where $v_{j}$ is the ion-neutral collision frequency of the $j$-th ion.

Poisson equation:

$\nabla^{2} \varphi=\frac{e}{\varepsilon_{0}}\left(n_{\mathrm{e}}-n_{1}-n_{2}\right)$.

In addition, the quasi-neutral condition at the sheath edge leads to

$n_{\mathrm{e} 0}-n_{10}-n_{20}=0$.

These equations are completed by Boltzmann relation for electron density. Compared with ions, the electrons would be accelerated to high energy very quickly as they are light and very mobile. Hence, they leave behind a large ion charge and so forces on them are closely in balance. Neglecting the drift terms in the force balance equation and considering the limit of $m_{\mathrm{e}} \rightarrow 0$ leads to Boltzmann relation for the electrons as follows [53]:

$n_{\mathrm{e}}=n_{\mathrm{e} 0} \exp \left(e \varphi / k_{\mathrm{B}} T_{\mathrm{e}}\right)$.

In the next section, we solve these basic equations numerically to study the sheath dynamics. For numerical solving of the equations, we assume that the plasma sheath variables vary only in the $z$-direction. Based upon this assumption, the dimensionless forms of the plasma sheath equations are as follows:

$N_{\mathrm{e}}=\exp (-\eta)$

$N_{1} u_{1 z}=\frac{M_{1}}{1+\delta}, \quad N_{2} u_{2 z}=\frac{\delta M_{2}}{1+\delta}$,

$u_{1 z} \partial_{\xi} u_{1 x}=\gamma_{1} \cos \theta u_{1 y}-\alpha_{1} u_{1}^{p_{1}+1} u_{1 x}$,

$u_{1 z} \partial_{\xi} u_{1 y}=\gamma_{1} \sin \theta u_{1 z}-\gamma_{1} \cos \theta u_{1 x}-\alpha_{1} u_{1}^{p_{1}+1} u_{1 y}$,

$u_{1 z} \partial_{\xi} u_{1 z}=\partial_{\xi} \eta-\gamma_{1} \sin \theta u_{1 y}-\alpha_{1} u_{1}^{p_{1}+1} u_{1 z}$,

$u_{2 z} \partial_{\xi} u_{2 x}=\mu \gamma_{2} \cos \theta u_{2 y}-\mu^{-p_{2}} \alpha_{2} u_{2}^{p_{2}+1} u_{2 x}$,

$u_{2 z} \partial_{\xi} u_{2 y}=\mu \gamma_{2} \sin \theta u_{2 z}-\mu \gamma_{2} \cos \theta u_{2 x}-\mu^{-p_{2}} \alpha_{2} u_{2}^{p_{2}+1} u_{2 y}$,

$u_{2 z} \partial_{\xi} u_{2 z}=\mu^{2} \partial_{\xi} \eta-\mu \gamma_{2} \sin \theta u_{2 y}-\mu^{-p_{2}} \alpha_{2} u_{2}^{p_{2}+1} u_{2 z}$, $\partial_{\xi}^{2} \eta=N_{1}+N_{2}-N_{\mathrm{e}}$

where $M_{j}=u_{\mathrm{jz} 0}=v_{\mathrm{jz} o} / c_{s 1}, \delta=n_{20} / n_{10}, \quad \mu=\left(m_{1} / m_{2}\right)^{0.5}$, $\gamma_{j}=\left(\varepsilon_{0} / n_{\mathrm{e} 0} m_{j}\right)^{0.5} B_{0}$ and $\alpha_{j}=n_{\mathrm{n}} \lambda_{\mathrm{D}} \sigma_{\mathrm{sj}}$ for $j=1$ and $2 . \alpha$ and $\gamma_{2}=\mu \gamma_{1}$ are the dimensionless parameters that specify the strength of the drag and magnetic forces in the plasma sheath equations, respectively. Also, $p_{j}$ is the $p$ value for the $j$-th ion (see Eqs. 1 and 2).

\section{Numerical results and discussion}

This section includes the numerical solution of the normalized equations and discussion on the sheath dynamics for different dependency of collision frequency on the ions velocities. At first, we use a numerical example for investigating the effect of collisional regime on plasma sheath dynamics. The dimensionless parameters in our numerical example are as follows: $\mu=0.1, \alpha_{1}=0.15$, $\alpha_{2}=0.45, \delta=0.1, \gamma_{1}=5$ and $\theta=40^{\circ}$. Also, the boundary values have been considered as $\phi_{0}=0$, $u_{10}=(0.3,0.3,1)$ and $\boldsymbol{u}_{20}=(0.1,0.1,1)$ and the electrostatic field at the sheath edge is taken to be an infinitesimal value to prevent the divergence of the numerical results. The boundary values of the ion velocity have been adopted, so that the Bohm criterion in the multicomponent plasma is satisfied $[8,54]$. Considering the nonzero components of the velocities parallel to the wall enables us to investigate the oblique entrance of the ions into the sheath.

Figure 1 shows the components of the velocity of lighter ion for constant mean free path $\left(p_{1}=0\right)$ and constant collision frequency $\left(p_{1}=-1\right)$.

The figure shows the ion gyration movement because of the external magnetic field. As it is seen, the ion gyro radius decreases by increasing the distance from the sheath edge. Also, the decrease of ion gyro radius for $p_{1}=-1$ is smaller than that of $p_{1}=0$. In other words, the drag force diminishes the helical movement of the lighter ion, and the diminution of the ion helical movement for constant mean free path is much stronger than that of constant collision frequency. It means that the drag force conquers the magnetic force quickly in constant collision mean free path regime, but it dominates slowly in constant collision frequency regime. These effects can be investigated by studying the effect of ' $p$ ' value on the fluctuations of the ion flow velocity in depth direction.

Figure 2 shows the velocity of lighter ion in the $z$-direction versus the distance from the sheath edge.

As seen, the amplitude of the fluctuations of the ion velocity decreases by penetrating into the sheath and increasing the drag force. Also, the results show that the 
ion velocity decreases by increasing the $p_{1}$ value from -1 to 0 . From the results, the sheath dynamics for $p_{1}=-1$ is approximately the same as the collisionless magnetized plasma sheath. Our numerical results showed that the velocity of the lighter ion is approximately independent of the $p_{2}$ value. Therefore, in a multi-component plasma sheath where the density of the heavier ion is a small percent of the lighter ion, the dynamics of the lighter ion is independent of the collisional regime of the heavier ion. The characteristics of the lighter ion in the plasma sheath depend on its collisional regime. Results shows that the oscillations of the velocity of the lighter ion and their amplitudes depend on the collisional regime of the lighter

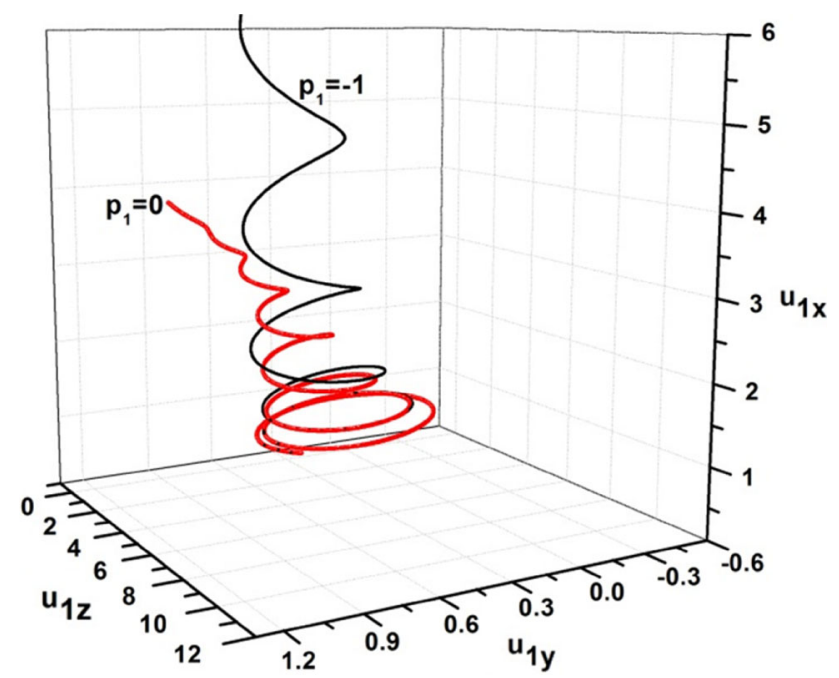

Fig. 1 The ion helical movement of the lighter ion in two collisional regimes: constant frequency $\left(p_{1}=-1\right)$ and constant mean free path $\left(p_{1}=0\right)$

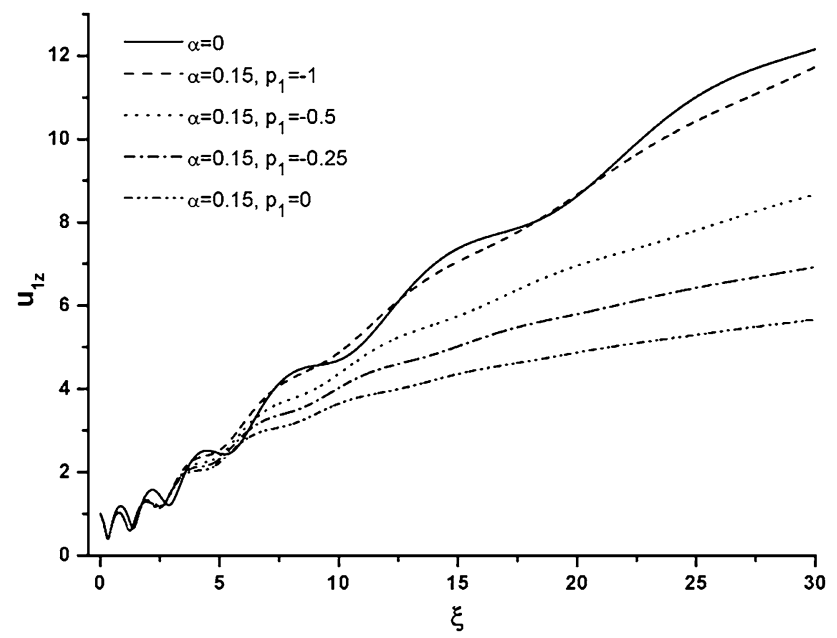

Fig. 2 The lighter ion velocity in the depth direction for different values of $p_{1}$ where $p_{2}=0$ ion: for low-pressure plasma, the plasma sheath dynamics is approximately the same as that of the collisionless plasma sheath; however, by increasing the pressure, the collisions between ions and stationary neutrals tend to damp the oscillations and gradually diminish their amplitudes. Thus, the effects of magnetic field on plasma increase if the law dependency of the collision regime is satisfied by $p$ values smaller than zero.

Figure 3 shows the effects of $p_{1}$ and $p_{2}$ values on the velocity of the heavier ion in the $z$-direction. From the results, $u_{2 z}$ increases by increasing the $p_{1}$ value (unlike the lighter ion velocity). It means that increasing the $p_{2}$ value tends to increase the collision effect and decrease the heavier ion velocity in the depth direction; however increasing the $p_{1}$ value tends to increase the heavier ion velocity. Nonetheless, as the effect of the collisional regime of heavier ion is stronger than that of the lighter ion, the ion velocity of the heavier ion decreases on increase of pressure.

Figure 4 indicates the effects of $p_{1}$ and $p_{2}$ values on the sheath electrostatic potential. As seen, the electrostatic potential decreases by decreasing the $p_{1}$ and $p_{2}$ values. The results show that the effect of the $p_{1}$ value is stronger than that of the $p_{2}$ value. The reason for this behavior can be explained by using the effect of the $p_{1}$ and $p_{2}$ values on the net positive space charge.

Figure 5 shows the net positive space charge versus the depth for different dependencies of ion collision frequency on the ion velocity. The fluctuations of the net positive space charge are due to the fluctuations of the lighter ion velocity in the $z$-direction, which leads to the fluctuations of the density distribution of the lighter ion. From the results, if the collisional regime of the plasma sheath is near to constant collision cross-sectional regime, the effects

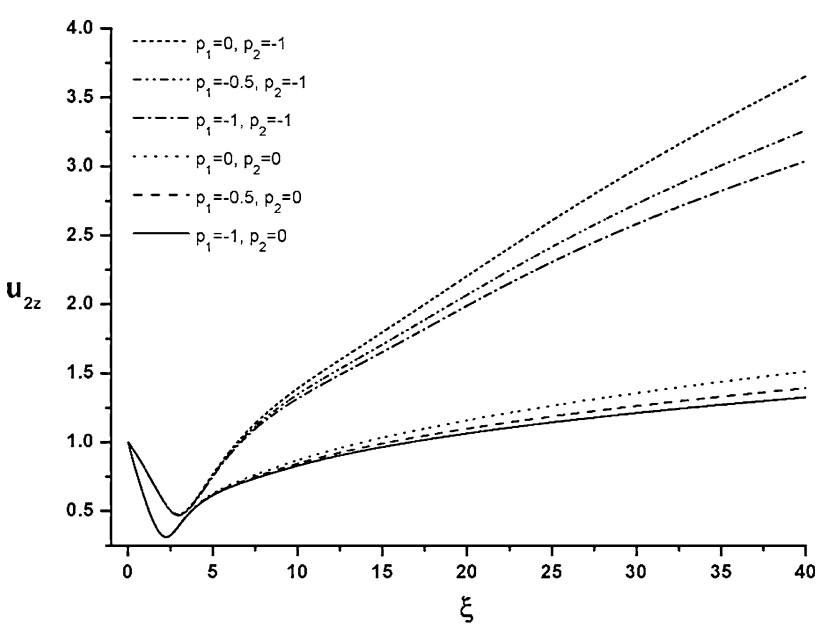

Fig. 3 The heavier ion velocity in the depth direction for different values of $p_{1}$ and $p_{2}$, where $\delta=0.1$ 


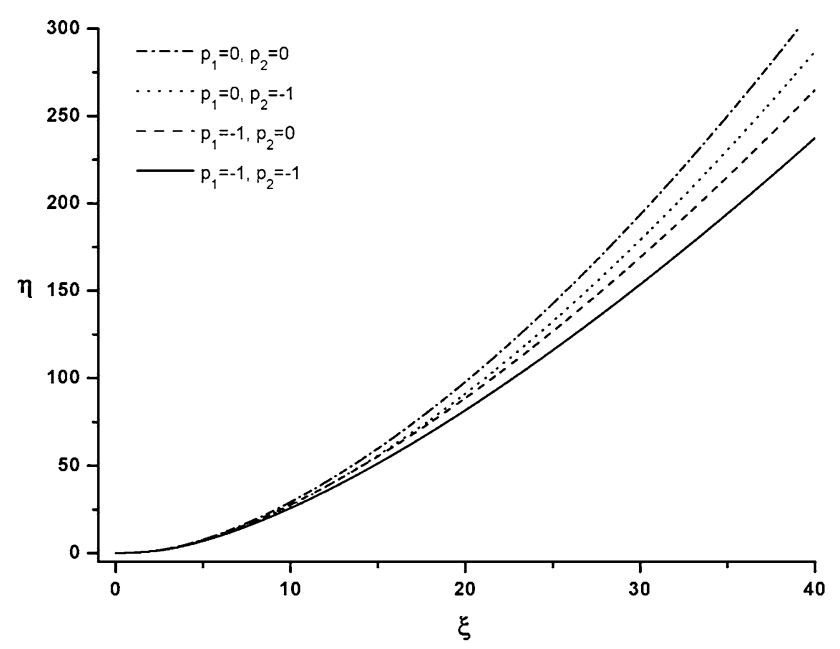

Fig. 4 The electrostatic potential versus the depth direction for different values of $p_{1}$ and $p_{2}$ where $\delta=0.1$

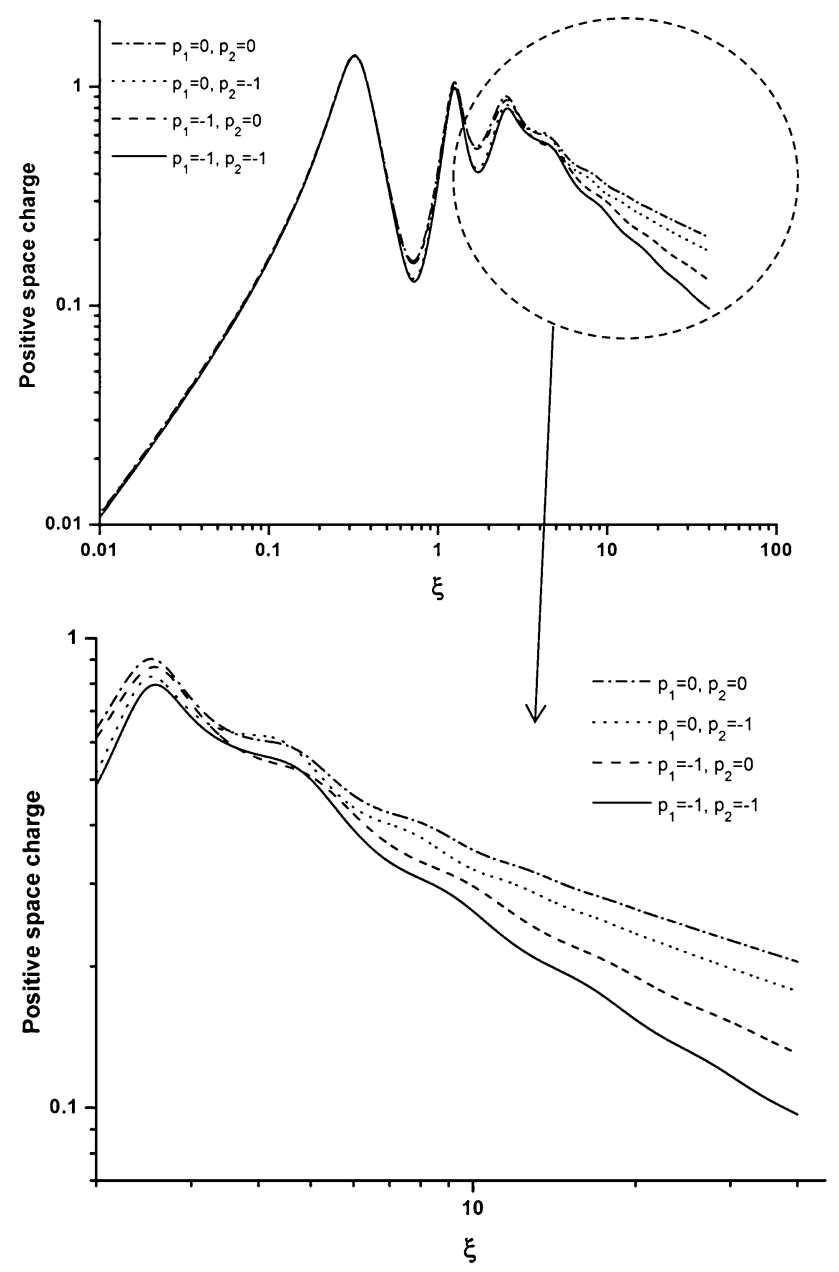

Fig. 5 The net positive space charge in the plasma sheath versus the depth direction for different dependencies of collision frequency on the ion velocity of collisions on the sheath structure increase (compared with constant collision frequency regime), which tends to decrease the ion velocity and gathering of ions. Also, the effect of the collisional regime of the lighter ion on the net positive space charge and electrostatic potential is more than that of the heavier ion.

The results of the numerical example show that the collisional regime between ions and neutrals has significant effects on plasma sheath dynamics, such as the energy and densities of the ions and the electrostatic potential. In the next subsection, we calculate the sheath dynamics of $\mathrm{Ar}^{+}-$ $\mathrm{He}^{+}$plasma considering the real collisional regime and compare the results with specific cases: constant collision cross section and constant collision frequency.

\section{Specific example: He-Ar plasma}

Consider a multi-component plasma containing two ion species, $\mathrm{Ar}^{+}-\mathrm{He}^{+}$, with the same density. Here, $m_{1}=4$ and $m_{2}=40$, so $\mu=0.1$. Also, assume that the plasma is in $\mathrm{B}=4 \mathrm{mT}$ external magnetic field, making an angle of $\theta=40^{\circ}$ with respect to the depth direction, neutral gas density $n_{\mathrm{g}}=3 \times 10^{14} \mathrm{~cm}^{-3}$ in $10 \mathrm{mTorr}$ pressure at room temperature $\mathrm{KT}=0.02 \mathrm{eV}$, electron density $n_{\mathrm{e} 0}=10^{9}$ $\mathrm{cm}^{-3}$ and $\sigma_{2 s}=3 \sigma_{1 s}=5 \times 10^{-15} \mathrm{~cm}^{2}$ [41]. Therefore, the dimensionless parameters are $\gamma_{1}=5$ and $\alpha_{1}=\alpha_{2} / 3=0.15$. For argon in the range of $3-300 \mathrm{eV}$, the power law is most closely satisfied by -0.25 , so $p_{2}=-0.25$. However, for helium ions streaming through a low-pressure plasma sheath, the cross section is more closely approximated by the constant mean free path, $p_{1}=0$. Figure 6 shows the normalized kinetic energy of argon ions and compares the result with the results of Ref. [33] in which $p_{1}=p_{2}=0$. The result shows that the argon ion energy is not the same as the constant collision cross-sectional regime. Consider that the most closely satisfied $p_{2}$ value for argon ion tends to increase of the energies of argon ions and small decrease of that of helium ions. As the results show, the difference between the real collisional regime and constant collision cross-sectional regime increases by increase of the ions' distance from the sheath edge. Therefore, the most closely satisfied dependency of collision cross section to the ion velocity should be considered in the investigation of the multi-component plasma sheath.

\section{Conclusions}

We studied the sheath dynamics containing two positive cold ions in the presence of an external magnetic field. We considered a collisional plasma sheath where the collision frequencies of both ions have a power law dependency on the ions' flow speeds. The numerical results showed that 

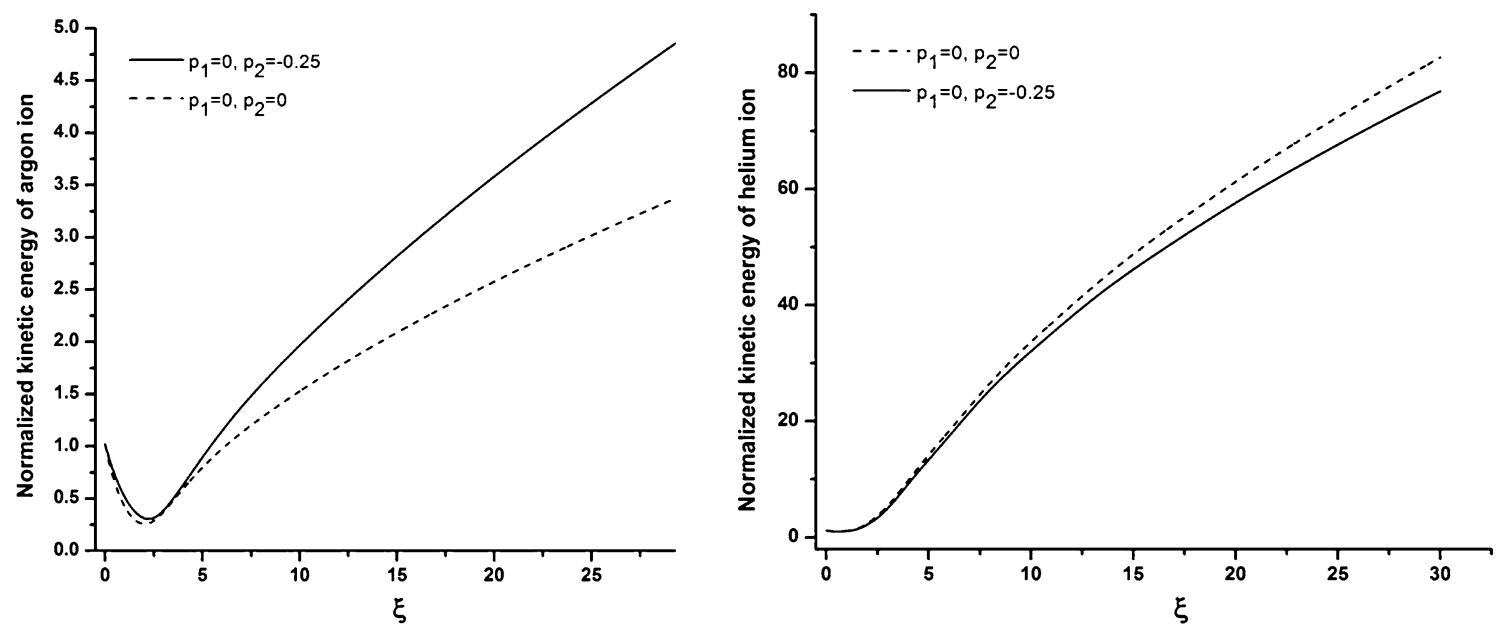

Fig. 6 Normalized kinetic energy of argon and helium ions in the $\mathrm{Ar}^{+}-\mathrm{He}^{+}$plasma sheath where the densities of the ions are the same

near the sheath edge, the sheath dynamics are independent of the collision regime. However, by increasing the distance from the sheath edge and increasing the ions' velocity in the cathode direction, the effects of the collision regime on the sheath dynamics are as follows:

- The velocity of the lighter ion in the depth direction decreases by increasing the $p_{1}$ value and is independent of the $p_{2}$ value. In other words, the maximum effect of drag force on the lighter ion velocity corresponds to the case where the lighter ion is in the constant collisional mean free path regime.

- Maximum effect of drag force on the heavier ion velocity corresponds to $p_{1}=-1$ and $p_{2}=0$.

- The effect of collision regime of lighter (heavier) ion on some sheath dynamics such as electrostatic potential and net positive space charge is more than that of heavier (lighter) ion for $\delta<1(\delta>1)$.

- When the occupation fraction of the heavier and lighter ions in the sheath is approximately the same $(\delta \sim 1)$, two ions can be considered as two independent ions. Hence, the maximum effect of drag force on the sheath dynamics corresponds to the case that both ions are in the constant mean free path regime $\left(p_{1}=p_{2}=0\right)$. Also, the effect of the collisional regime of the heavier ion on the sheath dynamics increases by increasing the $\delta$ value.

- The sheath dynamics is approximately the same as collisionless magnetized plasma sheath when the collision frequencies of the heavier and lighter ions are constant.

Open Access This article is distributed under the terms of the Creative Commons Attribution 4.0 International License (http://crea tivecommons.org/licenses/by/4.0/), which permits unrestricted use, distribution, and reproduction in any medium, provided you give appropriate credit to the original author(s) and the source, provide a link to the Creative Commons license, and indicate if changes were made.

\section{References}

1. Riemann, K.U.: J. Phys. D Appl. Phys. 24, 493-518 (1991)

2. Franklin, R.N.: J. Phys. D Appl. Phys. 36, R309-R320 (2003)

3. Riemann, K.U., Seebacher, J., Tskhakaya Sr, D.D., Kuhn, S.: Plasma Phys. Control. Fusion 47, 1949 (2005)

4. Franklin, R.N.: J. Phys. D 37, 1342 (2004)

5. Riemann, K.U.: Plasma Sour. Sci. Technol. 18, 014006 (2009)

6. Vranjes, J., Pandey, B.P., Tanaka, M.Y., Poedts, S.: Phys. Plasmas 15, 123505 (2008)

7. Stokes, J.D.E., Samarian, A.A., Vladimirov, S.V.: Phys. Rev. E 78, $036402(2008)$

8. Masoudi, S.F., Jafari, Gh, Shorakaee, H.: Vacuum 83, 1031-1035 (2009)

9. Khoramabadi, M., Ghomi, H., Kant Shukla, P.: J. Appl. Phys. 109, 073307 (2011)

10. Cheung, N.W.: Mater. Chem. Phys. 46, 132 (1996)

11. Conrad, J.R.: J. Appl. Phys. 62, 777 (1987)

12. Anders, A.: Handbook of Plasma Immersion Ion Implantation. Wiley, New York (2000)

13. Flender, U., Wiesemann-K, K.: Plasma Chem. Plasma Process. 15, 123 (1995)

14. Donnelly, V.M., Kornblit, A.: J. Vaccum Sci. Tech. A 31, 050825 (2013)

15. Lan, Ch., Peng, Y.F., Yang, Z., Long, J.D.: Plasma Sci. Technol 15, 945 (2013)

16. Chao-Hui, L., Yu-Fei, P., Zhen, Y., Ji-Dong, L.: Chins. Phys. C 37, 057002 (2013)

17. Han, Jeon G.: J. Phys. D Appl. Phys. 42, 043001 (2009)

18. Yang, Y.Z., Kim, K.H., Ong, J.L.: Biomaterials 26, 327-337 (2005)

19. Stamate, E., Sugai, H.: Phys. Rev. Lett. 94, 125004 (2005)

20. Stamate, E.: Plasma Phys. Control. Fusion 54, 124048 (2012)

21. Kim, G.H., Hershkowitz, N., Diebold, D.A., Cho, M.-H.: Phys. Plasmas 2, 3222 (1995)

22. Chengsen, L., Dezhen, W.: Surf. Coat. Technol. 171, 119 (2003)

23. Sheridan, T.E., Alport, M.J.: Appl. Phys. Lett. 64, 1783 (1994)

24. Pandey, B.P., Samarian, A., Vladimirov, S.V.: Plasma Phys. Control. Fusion 50, 10 (2008) 
25. Kostov, K.G., Barrosob, J.J., Ueda, M.: Surf. Coat. Technol. 201, 8398 (2007)

26. Franklin, R.N.: J. Phys. D Appl. Phys. 36, 1806 (2003)

27. Valentini, H.B., Herrmann, F.: J. Phys. D 29, 1175 (1996)

28. Benilov, M.S.: J. Phys. D 29, 364 (1996)

29. Franklin, R.N.: Plasma Sources Sci. Technol. 10, 162 (2001)

30. Severn, G., Wang, X., Ko, E., Hershkowitz, N.: Phys. Rev. Lett. 90, 145001 (2003)

31. Franklin, R.N.: J. Phys. D 36, 34 (2003)

32. Hatami, M.M., Niknam, A.R., Shokri, B., Ghomi, H.: Phys. Plasmas 15, 053508 (2008)

33. Hatami, M.M., Shokri, B., Niknam, A.R.: J. Phys. D Appl. Phys. 42, 025204 (2009)

34. Hala, M.A., Hershkowitz, N.: Rev. Sci. Instrum. 72, 2279 (2001)

35. Severn, G.D., Wang, X., Ko, E., Hershkowitz, N.: Phys. Rev. Lett. 90, 145001 (2003)

36. Lee, D., Severn, G., Oksuz, L., Hershkowitz, N.: J. Phys. D Appl. Phys. 39, 5230-5235 (2006)

37. Lee, Dongsoo, Hershkowitz, Noah, Severn, Greg D.: Appl. Phys. Lett. 91, 041505 (2007)

38. Khoramabadi, M., Ghomi, H., Shukla, P.K.: J. Plasma Phys. 79, 267-271 (2013)

39. Shaw, A.K., Kar, S., Goswami, K.S.: Phys. Plasmas 19, 102108 (2012)
40. Masoudi, S.F.: J. Phys. D Appl. Phys. 40, 6641-6645 (2007)

41. Lieberman, M.A., Lichtenberg, A.J.: Principle of Plasma Discharges and Materials Processing, 2nd edn. Wiley, Hoboken (2005)

42. Sheridan, T.E., Goree, J.: Phys. Fluids B 3, 2796 (1991)

43. Liu, J., Wang, Z., Wang, X.: Phys. Plasmas 10, 3032 (2003)

44. Yasserian, K., Aslaninejad, M.: Eur. Phys. J. D 67, 161 (2013)

45. Moulick, R., Mahanta, M.K., Goswami, K.S.: Phys. Plasmas 20, 094501 (2013)

46. Masoudi, S.F.: Vacuum 81, 871-874 (2007)

47. Hatami, M.M.: Phys. Plasmas 20(1), 013509 (2013)

48. Yankun, J., Xiu, Z., Huiping, L., Minghui, Q.: Plasma Sci. Technol. 13(5), 519 (2011)

49. Zou, X., Qiu, M., Liu, H., Zhang, L., Liu, J., Gong, Y.: Vacuum 83(1), 205-208 (2009)

50. Gan, Bao-Xia, Chen, Yin-Hua: Chin. Phys. Lett. 24(7), 2003-2005 (2007)

51. Shaw, A.K., Kar, S., Goswami, K.S.: Phys. Plasmas 19(10), 102108 (2012)

52. Yasserian, K., Aslaninejad, M.: Phys. Plasmas 19(7), 073507 (2012)

53. Chen, F.: Introduction to Plasma Physics and Controlled Fusion. Plenum, New York (1984)

54. Riemann, K.-U.: IEEE Trans. Plasma Sci. 23, 709 (1995) 